\title{
Pathogenic fungi-induced susceptibility is mitigated by mutual Lactobacillus plantarum in the Drosophila melanogaster model
}

\author{
Wanzhen $\mathrm{Su}^{1+}$, Jialin $\mathrm{Liu}^{1+}$, Peng Bai ${ }^{2 \dagger}$, Baocang $\mathrm{Ma}^{1}$ and Wei $\mathrm{Li}^{3^{3}}$ (D)
}

\begin{abstract}
Background: Since animals frequently encounter a variety of harmful fungi in nature, their ability to develop sophisticated anti-fungal strategies allows them to flourish across the globe. Extensive studies have highlighted the significant involvement of indigenous microbial communities in human health. However, the daunting diversity of mammalian microbiota and host genetic complexity are major obstacles to our understanding of these intricate links between microbiota components, host immune genotype, and disease phenotype. In this study, we sought to develop a bacterium-fungus-Drosophila model to systematically evaluate the anti-fungal effects of commensal bacteria.

Results: We isolated the pathogenic fungal strain, Diaporthe FY, which was detrimental to the survival and development of Drosophila upon infection. Using Drosophila as a model system, Drosophila-associated Lactobacillus plantarum functioned as a probiotic, and protected the flies from mortality induced by Diaporthe FY. Our results show that L. plantarum hindered the growth of Diaporthe FY in vitro, and decreased the mortality rate of Diaporthe FY-infected flies in vivo, consequently mitigating the toxicity of Diaporthe FY to the hosts. Additionally, the presence of L. plantarum overrode the avoidance of oviposition on Diaporthe FY-associated substrates.
\end{abstract}

Conclusions: Diaporthe FY was identified as a potential Drosophila pathogen. Commensal L. plantarum mitigated the susceptibility of Drosophila to pathogenic fungi, providing insight into the natural interplay between commensal and pathogenic microbial communities that contribute to animal health and pathogenesis.

Keywords: L. plantarum, Fungal infection, Drosophila, Antagonist, Oviposition

\section{Background}

Metazoans harbor a plethora of indigenous microbes (collectively referred to as the microbiota) that routinely influence the physiology and fitness of their host $[1,2]$, while in turn, the hosts shape the gut microbiota. This forging symbiosis enables the hosts to outcompete a variety of pathogens in the environment. In fact, several host phenotypes are shaped largely by the combination of the genome and microbiome [3, 4]. Consequently, commensals are critically linked to host fitness, including development,

\footnotetext{
* Correspondence: liuwei@sxmu.edu.cn

+Wanzhen Su, Jialin Liu and Peng Bai contributed equally to this work. ${ }^{3}$ Department of Medical Laboratory Science, Shanxi Medical University Fenyang College, Fenyang 032200, Shanxi, China

Full list of author information is available at the end of the article
}

fecundity, and lifespan. However, host phenotypes have been traditionally assessed in the context of their microbiota, with little attention devoted to effects of the microbiota on host fitness. In particular, the underlying mechanisms by which the microbiota assist their hosts in combating pathogens remain largely undefined.

In the wild, Drosophila mainly feed and breed on rotting fruits that are inhabited by both mutualistic and antagonistic microbes $[5,6]$. Due to their saprophagous foraging behavior, Drosophila ingest many potentially pathogenic fungi from either food resources or the surrounding environment [7, 8]. Although most microbes are not pathogenic [9], pathogens indeed engender the occurrence and severity of infection in the fly. Antagonistic fungi generate

(c) The Author(s). 2019 Open Access This article is distributed under the terms of the Creative Commons Attribution 4.0 International License (http://creativecommons.org/licenses/by/4.0/), which permits unrestricted use, distribution, and 
an astonishing variety of secondary metabolites that threaten insect life [10-12]. In addition, plant thorn injury and ectoparasitic mite biting frequently result in cuticle breaches, which aggravates the fungal infection. However, flies fundamentally employ antifungal strategies to flourish in the wild [7]. Although extensive studies have revealed that the microbiota promote the immune response to pathogenic fungi development and restrict pathogen colonization $[2,13,14]$, the role of specific bacterial species and/or strains in combating pathogens remain poorly understood. Therefore, there is a need for a model organism to examine the intricate interconnection between hosts, commensals, and pathogens.

Drosophila frequently acquires commensals through plant food, and provides amenable insight into the mechanisms by which commensals outcompete pathogens, due to genetic tractability and the ease of generating gnotobiotic animals $[15,16]$. The Lactobacillus genus is one of most common bacteria present in appreciable numbers in both mammals and Drosophila [6]. Moreover, studies have shown that $L$. plantarum fully recapitulates the beneficial effects of the complex microbiota, and influences several aspects of host physiology, including behavior, gut epithelial homeostasis, nutrition, and postembryonic development [13, 17-19]. Moreover, L. plantarum is required to protect flies against fly infection induced by food-borne bacteria (e.g., Pectobacterium carotovorum) [20]. Current molecular and genomic studies highlight the opportunities and challenges to uncover the interactions of entomopathogenic fungi and fly hosts. However, it remains unknown whether L. plantarum can protect hosts from pathogenic fungal infections.

To address these biological questions, we developed a Drosophila /bacterium/fungus ecological system that afforded the examination of commensal antagonism against pathogenic fungi. We found that commensal $L$. plantarum mitigated the pathogenic fungi-induced susceptibility of Drosophila, providing insight into the ecological significance that commensal bacteria may represent an integral contributor to Drosophila fitness upon infection.

\section{Results}

\section{Isolation and identification Drosophila-associated fungi}

A strain of Drosophila-associated fungus was isolated from fly food with mold in accordance with standard protocols. This strain was typically a filamentous fungus with white, velvety-like mycelia and dark grey conidial masses (Fig. 1a and b). The conidiophores were bi-verticillate with smooth-walled stipes, bearing short conidial chains (Fig. 1c). It promptly grew at the optimal temperature of $28^{\circ} \mathrm{C}-30^{\circ} \mathrm{C}$ and ramified the plates $(\Phi=90 \mathrm{~mm})$ within $48 \mathrm{~h}$. To confirm the reliability of the morphological identification, the strain was subjected to molecular identification based on an rDNA ITS sequence analysis. Based on the BLASTn search, it displayed $>99 \%$ similarity with a published sequence of Diaporthe $s p$. (identities $=555 / 558$ ) and was relatively close to other Diaporthe members. To distinguish our isolate from other strains, it was henceforth termed, Diaporthe FY. For taxonomic reconstruction, the other 12 sequences, including out-group species, were retrieved from GenBank to generate a phylogenetic tree (Fig. 1d). Diaporthe species are among the most frequent endophytes of a wide-range of plants, including grapevines $[21,22]$. Due to the saprophytic foraging behavior, flies might ingest Diaporthe from either food or surrounding resources.

\section{Diaporthe $\mathrm{FY}$ is a potential pathogen of $D$. melanogaster}

Drosophila frequently encounter a variety of commensal or pathogenic microbes in the wild. We first asked whether Diaporthe FY was beneficial or detrimental to Drosophila. To address this question, we examined the developmental timing and survival rate of the flies challenged with Diaporthe FY. Dechlorinated eggs were used to generate a specific interaction between hosts and specific microbes as previously described [23]. The results presented in Fig. 2a show that the hatched larvae rapidly succumbed to Diaporthe FY infection in vials containing more than $4 \times 10^{6} \mathrm{CFU}$ spores. The eclosion duration of the flies infected with Diaporthe FY was extended compared to conventionally reared (CR) flies (Fig. 2a). This finding implies that Diaporthe FY impeded the normal development of Drosophila. Consistent with a previous study [24], Drosophila was susceptible to Aspergillus flavus (Additional file 1), suggesting that the pathogenic fungus-induced morbidity of Drosophila could be generated in our laboratory. Intriguingly, the developmental time of adults challenged with less than $4 \times 10^{6} \mathrm{CFU}$ spores was shorter than that of germ free (GF) flies (Fig. 2a). This can be partially explained by the fact that the fungi could produce very low concentrations of toxic secondary metabolites during the exponential phase of nutritional growth; thus, its growth-promoting effects could override the inhibition of host development. Additionally, the survival of CR adults fed the Diaporthe FY molds was significantly lower than that of the mockinfected flies (Fig. 2b). Due to the causal relation between GF hosts and microbes, the survival of GF adults subjected to Diaporthe FY was also lower than that of their counterparts (Fig. 2b). These results suggest that Diaporthe FY diminished the relative survival of the flies. Moreover, innate immunity-associated genes were significantly triggered in the Diaporthe FY-infected flies compared to their counterparts (Fig. 2c), indicating that the flies developed a robust immune response to this invader. In agreement with a previous study [25], immune deficient PGRP-LC mutant flies were much more susceptible to Diaporthe FY than the wild-type flies (Fig. 2b), 


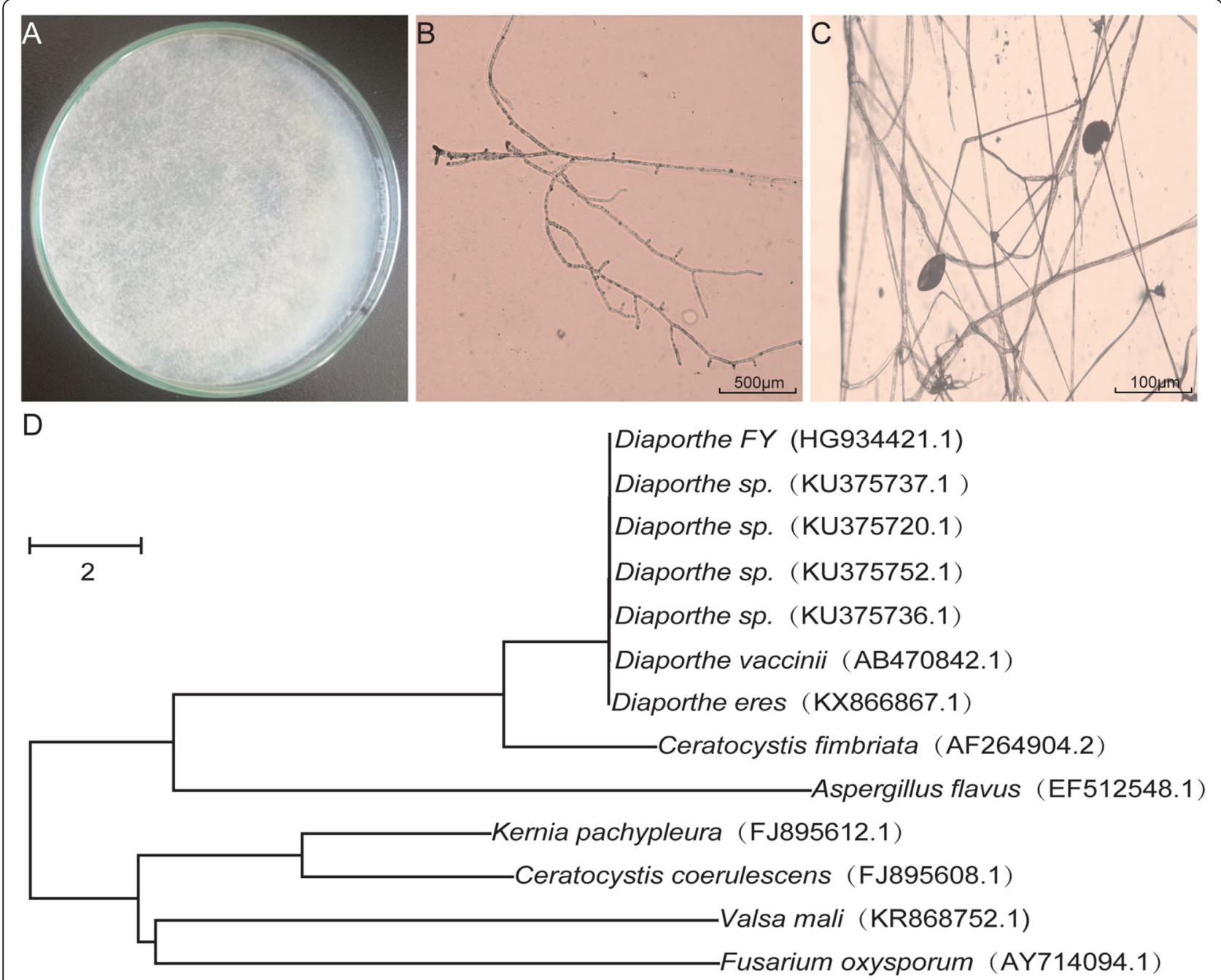

Fig. 1 Morphologies and phylogenetics of Diaporthe FY (a) Colony growth on the yeast-potato medium. Image depicts fungal development after $48 \mathrm{~h}$ incubation at $25^{\circ} \mathrm{C}$ on nutrient-rich medium. $\mathbf{b}$ and $\mathbf{c}$ Mycelia, conidiophores, and conidia of Diaporthe FY. $\mathbf{d}$ The phylogenetic tree of Diaporthe FY and its homologs constructed using the neighbor-joining method. Bar: Nucleotide divergence; numbers in the notes present bootstrap percentages; numbers in parentheses are the GenBank accession

indicating that Diaporthe FY was a potentially virulent pathogen towards $D$. melanogaster. Hence, we subsequently investigated the survival to septic injury by injecting Diaporthe FY spores into the body cavity of flies. Concomitantly, the flies challenged with septically infection were more likely to die compared to their corresponding control flies (Fig. 2d), indicating that Drosophila was susceptible to Diaporthe FY. Collectively, these results suggest that Diaporthe FY functions as a Drosophila-associated pathogen.

\section{L. plantarum undermines the susceptibility of Drosophila to Diaporthe FY infection}

Given that pathogenic fungal infections impose morbidity and mortality upon animals in the wild, it was proposed that the natural host microbiota could promote the survival of flies challenged with Diaporthe FY [26]. Previous studies have shown that $L$. plantarum, due to its vast metabolic repertoire, fostered host development by accelerating their growth rate [17]. We then examined the antifungal response of $L$. plantarum against Diaporthe FY by simultaneously inoculating them into sterilized Drosophila GF eggs. Indeed, supplementation with $L$. plantarum efficiently rescued the lethality of the Diaporthe FY-infected flies, as well as ameliorated the delay of pupa formation and adult eclosion (Fig. 2e and f). This result suggests that $L$. plantarum mitigated Drosophila susceptibility to Diaporthe FY.

\section{L. plantarum suppresses the growth of Diaporthe FY}

To confirm that L. plantarum competes with Diaporthe FY, we tested the inhibition of $L$. plantarum on the growth 


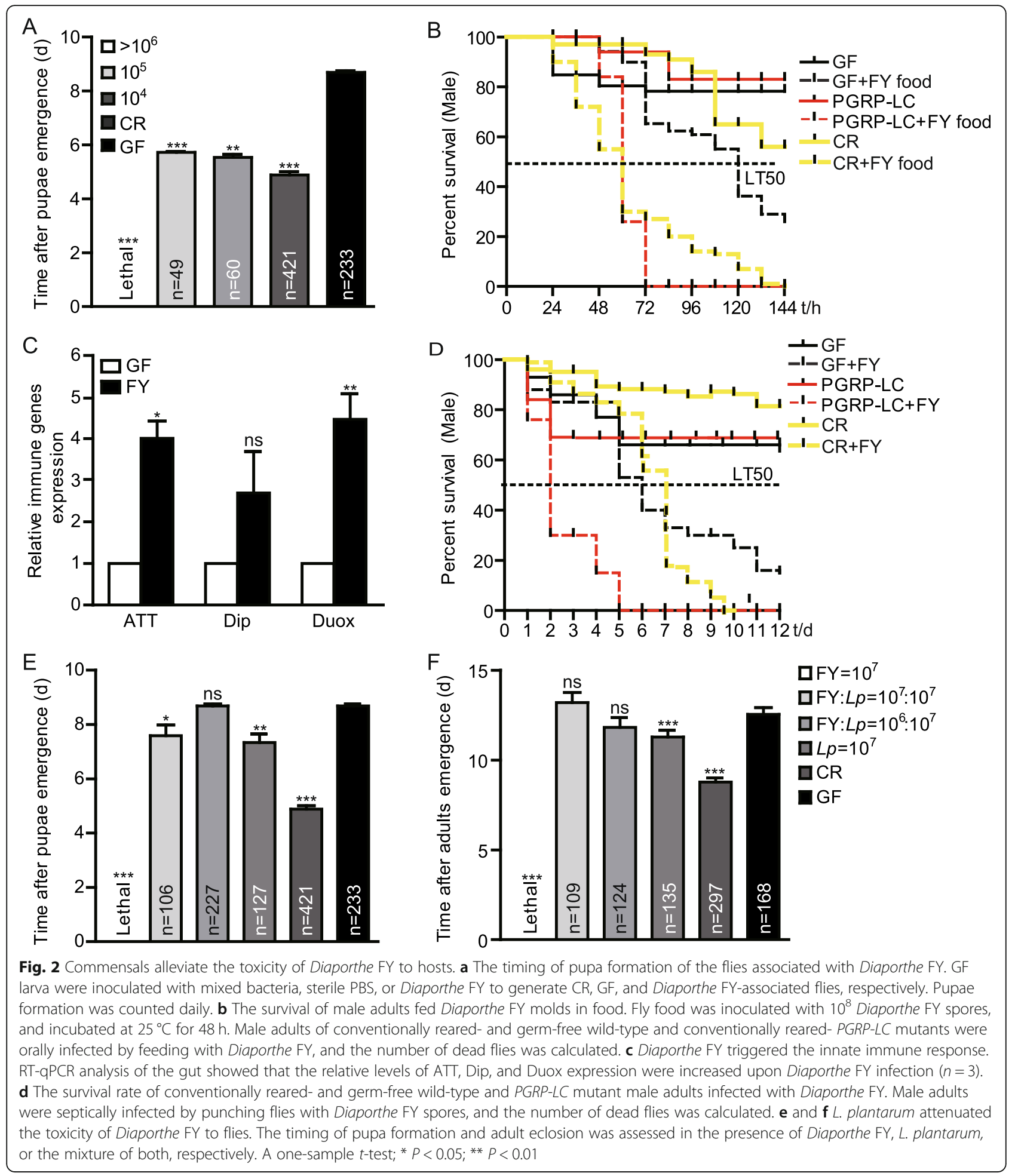

of Diaporthe FY in vitro. Our data showed that L. plantarum outcompeted Diaporthe FY in a dose-dependent manner (Fig. 3a). We quantified the suppressive effects by colony growing, mycelia branching, and spore forming assays. First, the colony growth of Diaporthe FY was decreased by L. plantarum compared to the control
(Fig. $3 b$ and c). Secondly, there were fewer mycelia in the presence of $L$. plantarum (Fig. $3 \mathrm{~d}$ ). In addition, the number of spores was dramatically decreased following L. plantarum inoculation (Fig. 3e). Taken together, these results suggest that $L$. plantarum potently reduced the survivability of Diaporthe FY. 

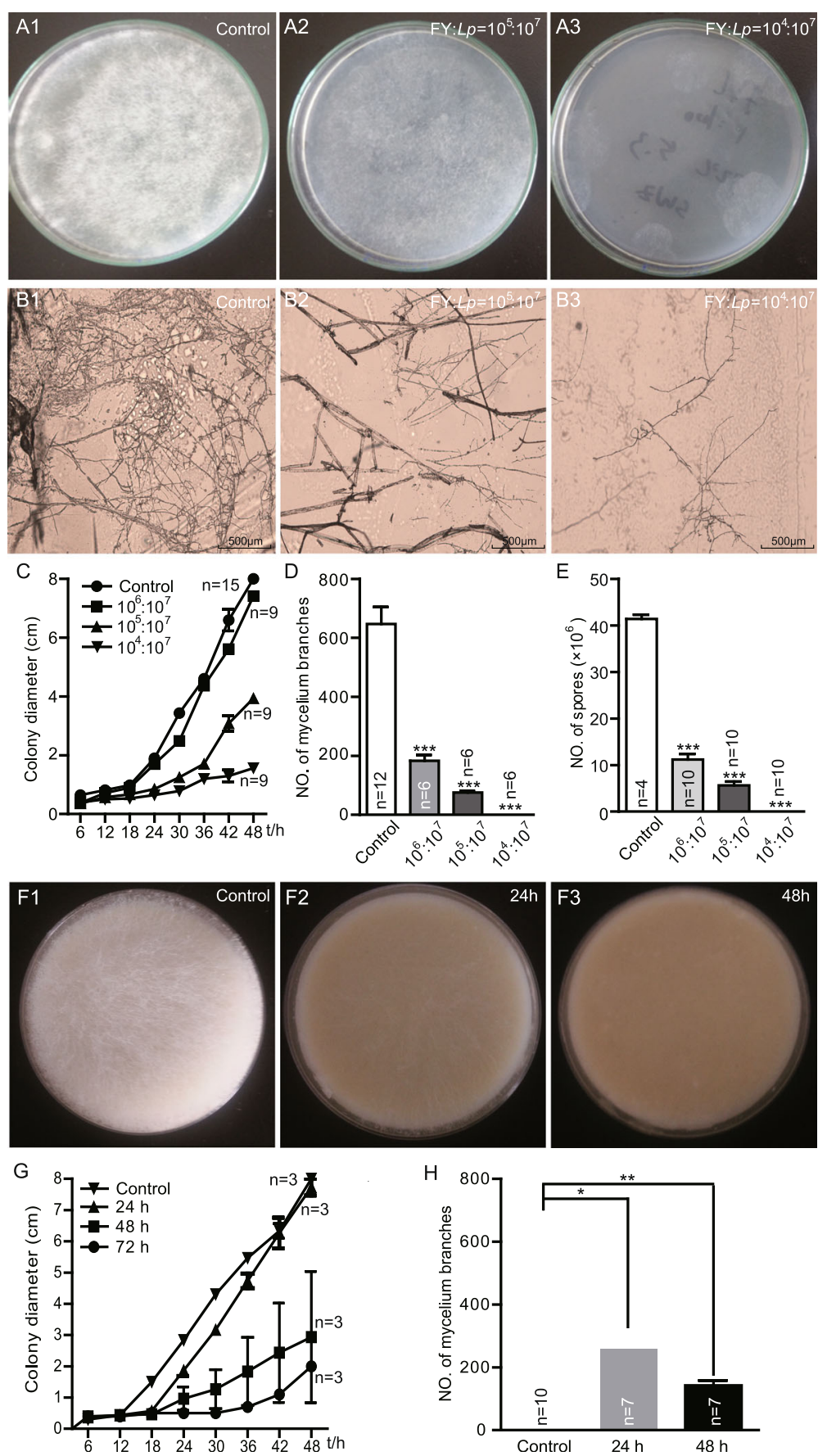

Fig. 3 L. plantarum hinders the growth of Diaporthe FY in vitro. a L. plantarum inhibited the growth of Diaporthe FY in a dose-dependent manner. Both Diaporthe FY and L. plantarum (at different ratios: A1, Diaporthe FY; A2, 1:100; A2, 1:1000) were simultaneously inoculated into nutrient rich medium and incubated at $25^{\circ} \mathrm{C}$ for $24 \mathrm{~h}$. The growth of Diaporthe FY is shown. $\mathbf{b}$ The growth of mycelia was inhibited in the case of L. plantarum. c-e The quantification of the colony growth rate, the number of mycelia, and spores of Diaporthe FY. $\mathbf{f}$ The growth of Diaporthe FY in fly food pre-incubated with L. plantarum. White mycelia were observed on the surface of the food (F1), whereas fewer mycelia were observed in L. plantarum-treated food (F2 and 3). $\mathbf{g}$ The colony growth and number of mycelium branches of Diaporthe FY in medium pre-incubated with L. plantarum. The one-sample $t$-test; ${ }^{*} P<0.05 ;{ }^{* *} P<0.01$

We further speculated that after dominating the niche, L. plantarum could thwart Diaporthe FY colonization. To this end, we pre-incubated the food with L. plantarum for different lengths of time $(24 \mathrm{~h}$, or $48 \mathrm{~h})$ and added Diaporthe FY to the "modified" diet. In agreement with the simultaneous competition, the growth of Diaporthe FY 
was also hindered when it was pre-inoculated into the food with $L$. plantarum. Of note, the longest incubation period completely inhibited the growth of Diaporthe FY (Fig. 3f). This inhibitory effect was further fortified by the decreased number of mycelia and spores (Fig. $3 g$ and h). Taken together, these findings support the L. plantarummediated inhibition of Diaporthe FY growth and dispersal.

\section{Lactic acid inhibits the growth of Diaporthe FY}

To further characterize the mechanism involved in $L$. plantarum-mediated inhibition of Diaporthe FY, we next sought to identify candidate inhibitory factors derived from L. plantarum metabolites. Lactic acid is generated by many lactic acid bacteria and exerts its antimicrobial effects by disrupting the cytoplasmic membrane or reducing the intracellular $\mathrm{pH}$ [27]. Since the strain of L. plantarum used in this study typically produced more than $75 \mathrm{mM}$ (approximately $0.7 \% \mathrm{w} / \mathrm{v}$ ) L-lactate at the end of fermentation (Additional file 2), we therefore focused on the role of L-lactate on inhibiting the growth of Diaporthe FY. To determine whether lactic acid could inhibit the growth of Diaporthe FY, we scored the fungal growth on a medium supplemented with different concentrations of L-lactate. The results showed that the growth of Diaporthe FY was inhibited in a L-lactate dose-dependent manner (Fig. 4a). Diaporthe FY was modestly inhibited by $0.5 \%$ lactic acid and robustly inhibited by $1 \%$ or higher doses of lactic acid. It was unlikely that this antifungal property was derived from the lower $\mathrm{pH}$ value, since the comparable $\mathrm{pH}$ values adjusted with $\mathrm{HCl}$ were unable to inhibit the growth of Diaporthe FY (Additional file 3). These data indicate that the inhibition of Diaporthe FY was partly attributed to the properties of lactic acid. The data further showed that the colony growth of lactic acid-treated Diaporthe FY was prominently decreased compared to the mockinfected flies (Fig. 4b). Likewise, the number of mycelia and hyphae were considerably lowered in the presence of lactic acid (Fig. 4c and d). Overall, our data suggest that lactate was an important factor that could inhibit the growth of Diaporthe FY.

\section{The synergism between Drosophila and L. plantarum to combat Diaporthe FY infection}

Upon pathogenic infection, Drosophila initiates an innate immune response through the production of reactive oxygen species and antimicrobial peptides. It was assumed that collaboration between the host and its commensals could more efficiently resist pathogenic fungi than either alone. To the end, the early third-instar larvae were seeded into the fly diet with Diaporthe FY and L. plantarum. Our data revealed that the colony growth of Diaporthe FY was significantly obstructed in the presence of larvae compared to that in the absence of larvae (Fig. 5a and c). This result indicated that Drosophila and commensals collaborated to antagonize pathogens. Similarly, the number of
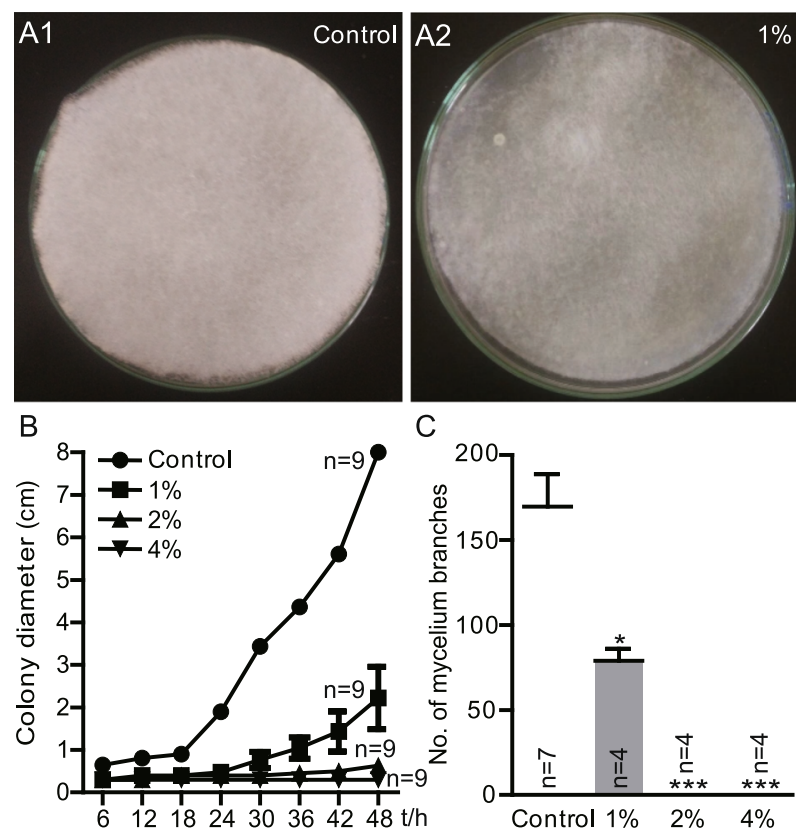

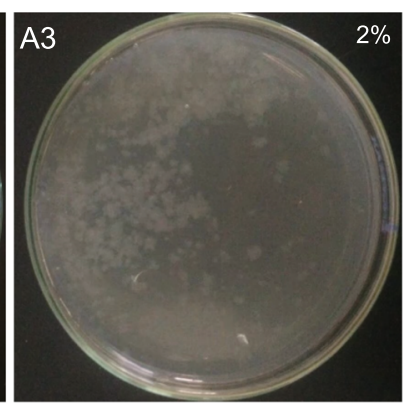

D

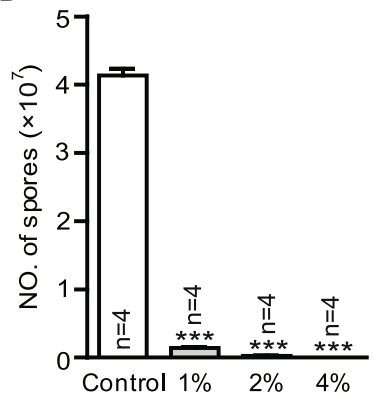

Fig. 4 Lactic acid exerts anti-fungal properties. a The growth of Diaporthe FY at different concentrations of lactic acid. Diaporthe FY was inoculated into nutrient-rich medium containing different concentrations of L-lactate, and incubated at $25^{\circ} \mathrm{C}$ for $48 \mathrm{~h}$. $\mathbf{b}$ The colony growth rate of Diaporthe FY treated with lactic acid. $\mathbf{c}$ and $\mathbf{d}$ The number of mycelia and spores in the case of lactic acid. The one-sample $t$-test; NS $P>0.05$; * $P<0.05$; ${ }^{* *} P<0.01$; ${ }^{* *} P<0.001$ 

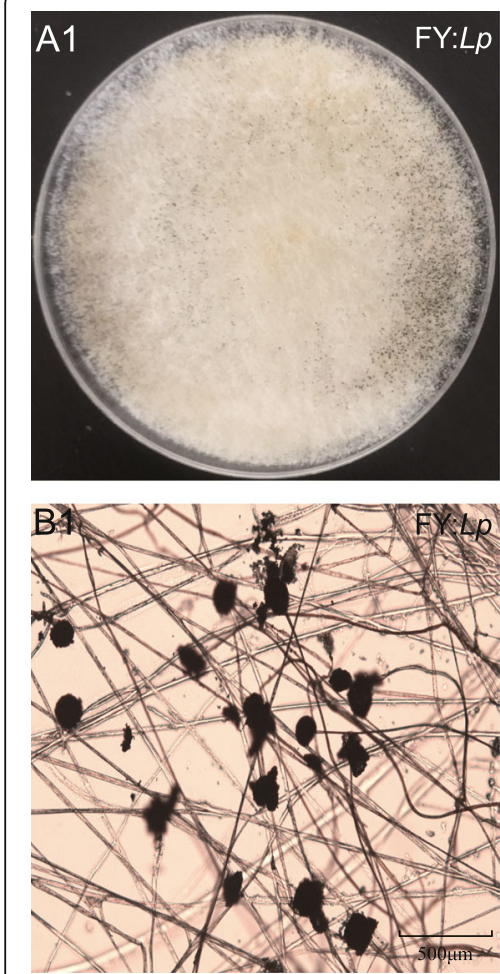

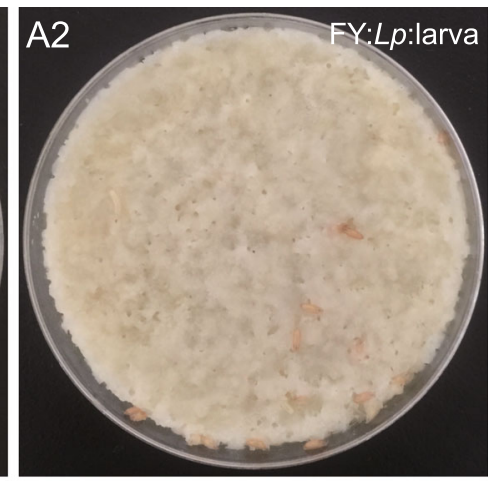

B2

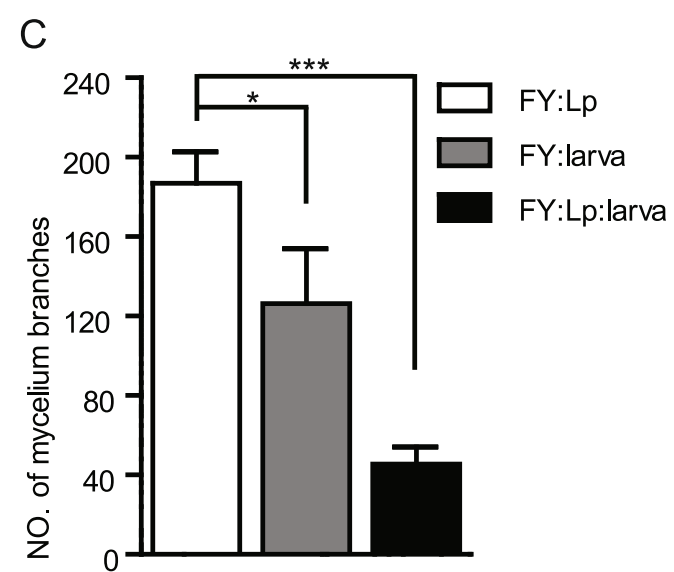

Fig. 5 Drosophila and L. plantarum synergize to defend against Diaporthe FY. a The colony growth of Diaporthe FY with L. plantarum in the presence or absence of larvae. A total of 25 third instar larva were transferred to each plate with a fly diet inoculated with Diaporthe FY, L. plantarum, or both. The plates were incubated at $25^{\circ} \mathrm{C}$ and colony growth was assessed at $72 \mathrm{~h}$. b Mycelia of Diaporthe FY with L. plantarum in the absence or presence of larvae. c The number of branching mycelia in the absence or presence of larvae. The one-sample $t$-test; $* * * ~ P<0.001$

branching mycelia was reduced in the presence of larvae compared to jn the absence of larvae (Fig. 5b and c). Intriguingly, Diaporthe FY did not form any spores in the case of larvae, partly due to the disrupted configuration of the hypha. These results demonstrate that Drosophila synergized with L. plantarum to suppress the growth of Diaporthe FY, which was critical for host survival against infection.

\section{L. plantarum reverses ovipositional avoidance to Diaporthe FY}

Using various sensory modalities, animals are able to swiftly respond to certain stimuli in their surrounding environment. To enhance the survival and fitness of their offspring, Drosophila females select favorable sites to deposit their eggs $[28,29]$. Our previous work showed that commensals (e.g., L. plantarum), elicited an oviposition preference of Drosophila using the two-choice assay [23]. Since Diaporthe FY imposed morbidity on both larval and adult Drosophila (Fig. 2), it would be reasonable to hypothesize that Drosophila could sense the presence of Diaporthe FY in potential egg-laying sites. As expected, female adults overwhelmingly avoided egglaying on the food treated with Diaporthe FY (Fig. 6a). Many molds produce an extraordinary range of secondary metabolites that repel insects [30, 31]. Indeed, the flies were robustly repulsed to laying their eggs on the surface of the halves containing metabolites of Diaporthe FY (Fig. 6b), which indicated that secondary metabolites of Diaporthe FY alerted the flies to the presence of toxic molds. We next wondered whether $L$. plantarum could alter the ovipositional repulsion of females to Diaporthe FY. As expected, the addition of L. plantarum dosedependently increased the oviposition index of the females, and even switched to laying eggs in fermented food with a predominance of L. plantarum (Fig. 6c), indicating that L. plantarum attenuated ovipositional avoidance to Diaporthe FY. We further asked whether L. plantarum could abolish the ovipositional aversion to Diaporthe FY when it had dominated the community. The diet was preincubated with $L$. plantarum for different lengths of time and then exposed to Diaporthe FY. We found that although the flies were aversive to ovipositing in fermented food pre-incubated with L. plantarum for $24 \mathrm{~h}$, this response was over-ridden in fermented food pre-incubated with L. plantarum for $48 \mathrm{~h}$ (Fig. 6d). Hence, our results demonstrated that commensals, if dominating the niche, significantly reversed the oviposition avoidance to pathogenic fungi. 

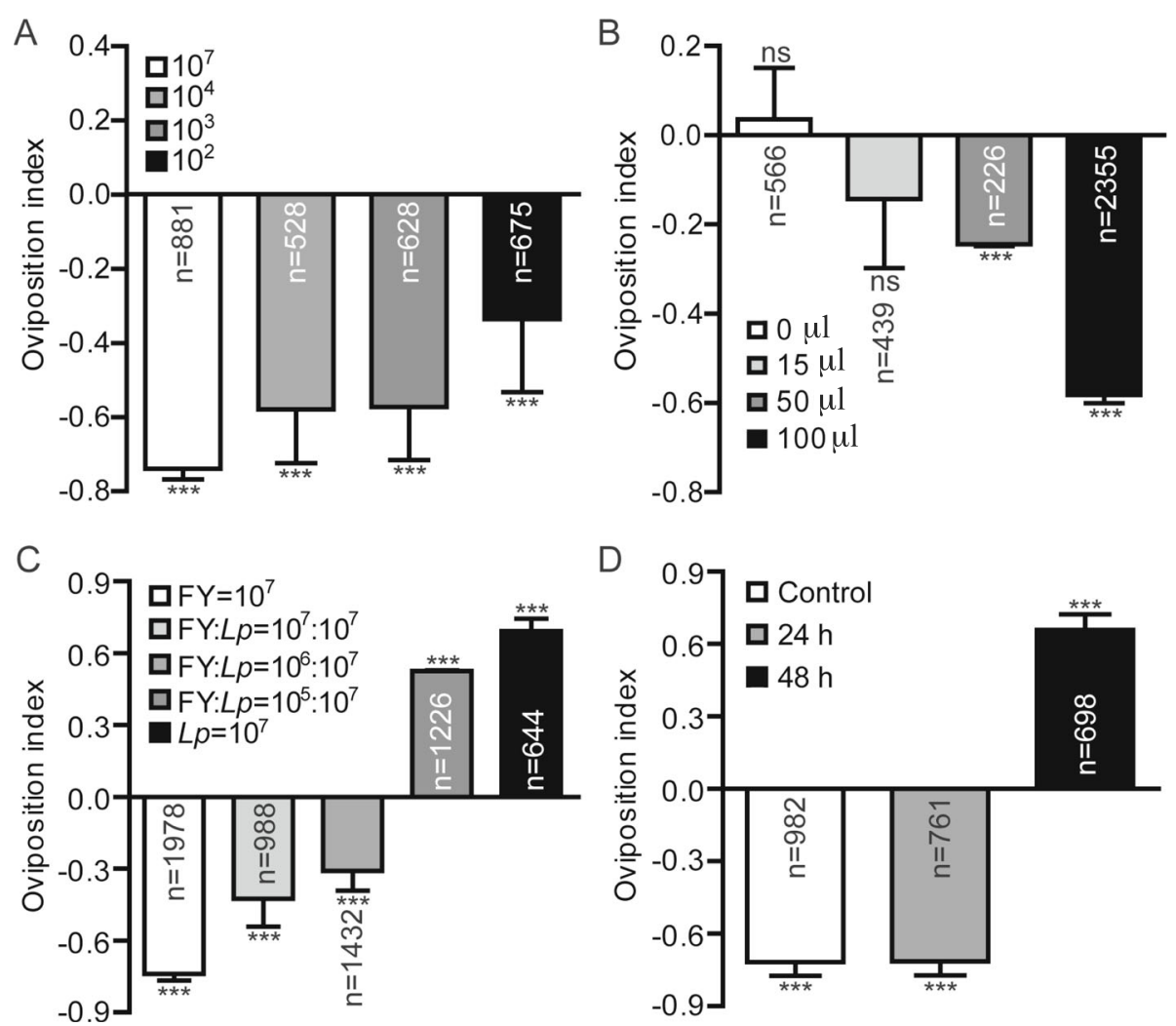

Fig. 6 L. plantarum prevented oviposition avoidance towards Diaporthe FY. a Quantification of egg-laying avoidance to diet fermented with Diaporthe FY. Egg-laying preference was assayed using a two-choice chamber. Mated females were transferred to the chamber and allowed to lay eggs for $8 \mathrm{~h}$. The number of eggs was counted on each half, and the oviposition preference was calculated. b Ovipositional avoidance towards metabolites of Diaporthe FY. The supernatant of liquid fly food was evenly distributed on the surface of halves in a dose-dependent manner. c L. plantarum reduced oviposition avoidance towards Diaporthe FY. d Oviposition preference for Diaporthe FY-treated diet that was previously inoculated with $L$. plantarum. The one-sample $t$-test was used to assess the mean deviance of each column from $0, n=6-14$; NS $P>$ $0.05 ;{ }^{*} P<0.05 ;{ }^{* *} P<0.01 ;{ }^{* *} P<0.001$

\section{Discussion}

Animals are colonized by abundant and diverse microbiota, which affect many aspects of host physiology and pathology [32, 33]. Despite advances in sequence-depended microbial profiling, little is known about the role of mutualistic microbes in antagonizing fungal pathogens in their host animals. This study has shown that Drosophila-associated commensal bacteria exhibited inhibitory capabilities against fungal infections. Moreover, commensal L. plantarum suppressed the growth of Diaporthe FY in vitro, and mitigated the fungal toxicity to Drosophila in vivo. In particular, $L$. plantarum predominantly overrode the egg-laying avoidance of Drosophila to Diaporthe FY. This integrative and synthetic community of Drosophila, bacterium, and fungus provides insight into the fundamental concepts and precise mechanisms involved in animal-commensal-pathogen interactions. The infection-associated Drosophila model used in this study was previously established by injecting a lethal dose of the pathogens into the body cavity of adult flies [34]. However, little attention has been devoted to complex bacterial context. In fact, a bacterial consortium approach that views the microbiome as a set of functional traits is likely to offer a more comprehensive means of protecting hosts from threatening pathogens. Consistently, the observed phenomenon supports our hypothesis that the protective traits conferred by the host's natural microbiota were naturally selected to enhance host survival in the context of a challenging environment rife with pathogens. Thus, our approach should facilitate the development of animal models that can better recapitulate complex natural pathological phenomena.

Entomopathogenic fungi play a pivotal role in regulating insect populations in nature [12]. These fungi have evolved highly diversified lifestyles, and are in competition with insects for natural resources. Metagenetic analysis has unraveled dozens of secondary metabolic gene clusters that encode an astonishing variety of secondary metabolites [10, 30, 31]. Although pathogenic fungi seriously threaten the survival of flies in nature, animals have developed fundamental antifungal strategies to thrive in the world. Drosophila possess an innate immune system that induces the production of antimicrobial peptides and reactive oxygen species to prevent 
fungal infection [35]. Alternatively, the mitigation of fungal toxicity can also be attributed to complex interactions between hosts and microbes. Commensals and/or probiotics outcompete pathogens through chemical inhibition, physical and nutritional competitive exclusion, and a variety of other adaptive mechanisms [36]. Lactic acid bacteria are widely considered to be natural antifungal microbes that can be found in fermented substrates [37, 38]. Lactic acid bacteria hamper the growth of many pathogenic fungi by inhibiting their adherence, establishment, replication, various pathogenic actions, and can also decompose mycotoxin to a certain extent [39]. In turn, larvae-derived maintenance factors enhance the propagation of this bacterial population, and override this cost of feeding and gut transit, forming an inextricable holobiont [26]. Notably, L. plantarum mon-associated and GF flies usually more resist against pathogens than CR ones. This is in part explained by the finding that CR flies are prone to dysbiosis with an increase in intestinal bacterium loading, and dramatic changes in the components and functions of the microbiota, which could aggravate the pathogenesis. More importantly, adult Drosophila function as a vector, and promote the ongoing dispersal of bacteria in the environment [40]. Therefore, the synergistic interaction between Drosophila and the microbiota exerts antifungal activity against a broad spectrum of molds in the wild.

While hosts exhibit behavioral-immune responses against pathogens, this important function remains underappreciated [41]. Female Drosophila possess the innate behavior of selecting favorable oviposition sites to increase the survivability of their offspring. Since larvae are vulnerable to predators due to their restricted mobility, selecting a favorite site to lay their eggs is an innate behavior of female. The hypothesis of 'mother-knows-best' ensures that female egglaying decisions have evolved to identify locations that will promote the greatest survival of offspring [42]. This is achieved by evaluating the nutritional and microbial content of potential oviposition sites. Previous studies, including our work, have shown a general theme that Drosophila are robustly allured to lay eggs in fermented food by commensal Lactococcus, Lactobacillus, Weissella, and Saccharomyces [23, 43]. However, they were vigorously repelled by the presence of harmful molds (e.g., Penicillium expansum) [44]. Thus, the females avoid laying eggs on the sites with fungal toxicants, which efficiently protects the hatched larvae from infection. Survival and reproduction strategies should be employed in the context of systemic ecology, in which flies balance the benefits and threats of commensals and pathogens, respectively. Therefore, Drosophila distinguishes commensals from pathogens and selects favorable sites for egg-laying. It is conceivable that females still switch to laying-eggs on fermented food when the nutrition of commensals overrides threats from pathogens. This is consistent with the observation that fermented food with $L$. plantarum and Diaporthe FY still attract females to lay eggs, partially because $L$. plantarum has dominated the niche and outcompeted Diaporthe FY.

Utilizing the Drosophila model system, we have revealed an ecological phenomenon whereby indigenous microbiota are required to defend Drosophila against pathogenic fungal infection. This model provides a reductionist approach for disentangling the inherent complexity of host-microbe interactions from the organismic to the molecular level. A more complete understanding of the underlying mechanism of the host and bacterial response to pathogens will facilitate the discovery of innovative probiotic interventions to foster the fitness of the microbe-host holobiont.

\section{Conclusion}

In this study, we identified and characterized Diaporthe FY as a potential Drosophila pathogen. Commensal $L$. plantarum is required and sufficient to ameliorate the susceptibility of Drosophila to Diaporthe FY by generating lactic acid. L. plantarum counteracted the avoidance of oviposition on Diaporthe $F Y$-associated substrates, which enhances the fitness of Drosophila offspring. In summary, our findings provide a first insight into the natural interplay between Drosophila, commensals and pathogens.

\section{Methods}

\section{Drosophila and microbe husbandry}

The Oregon R strain of D. melanogaster was used as wildtype flies. PGRP-LC mutant flies were kindly gifted by Dr. Zhai (Hunan Normal University, China). All flies were reared at $25^{\circ} \mathrm{C}, 60 \%$ humidity with a $12 \mathrm{~h} / 12 \mathrm{~h}$ light/dark cycle on standard cornmeal-yeast-sucrose food unless otherwise stated [23]. Drosophila was cultured with stand-

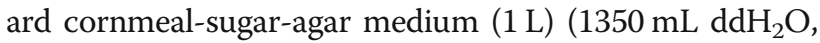
$13 \mathrm{~g}$ agar, $0.83 \mathrm{~g} \mathrm{CaCl}_{2}, 31.6 \mathrm{~g}$ sucrose, $63.2 \mathrm{~g}$ glucose, 77.7 $\mathrm{g}$ cornmeal, and $24 \mathrm{~g}$ yeast power) [28]. Fungi were cultured using Potato Dextrose Agar (PDA) medium at $25^{\circ} \mathrm{C}$. L. plantarum were cultured in selective medium De Man Rogosa Sharpe (MRS) at $35^{\circ} \mathrm{C}$. The mixture of Diaporthe $\mathrm{FY}$ and $L$. plantarum was cultured in MuellerHinton Agar (MHA) medium at $30^{\circ} \mathrm{C}$. L. plantarum was isolated from the gut of Drosophila with the Genbank accession number, KY038178. Aspergillus flavus (3.3950) was obtained from the China General Microbiological Culture Collection Center.

\section{Fungi isolation and identification}

Fungal strains were isolated from fly food with molds using PDA medium. Mycelium was briefly picked up, transferred to PDA medium, and incubated for $48 \mathrm{~h}$ at $25^{\circ} \mathrm{C}$. This procedure was repeated five times for purification. The fungus was grown on liquid PDA medium 
for $2 \mathrm{~d}$ at $25^{\circ} \mathrm{C}$. The mycelium was collected, and the genomic DNA was extracted and purified using a DNA isolation kit (Tiangen, Beijing, China). For identification, the internal transcribed spacer regions (ITS1 and ITS2) were amplified using PCR (Thermocycler, Germany) with the universal ITS primers, ITS1 $\left(5^{\prime}-\right.$ TCC GTA GGT GAA CCT GCGG-3') and ITS4 (5'-TCC TCC GCT TAT TGA TAT GC-3'). The PCR products were sequenced by a commercial company (Shenggong, Shanghai). The ITS DNA sequences were aligned against the nucleotide-nucleotide database (BLASTn) of the National Center for Biotechnology Information (NCBI) for the final identification of the isolates.

\section{Colony growth, branching mycelium, and sporulation assay}

A $1 \mu \mathrm{L}$ extract with $10^{6}$ spores was inoculated into MHA medium. To assess the effect of acid on fungi, L-lactate or chloric acid was directly added to the medium in a dosedependent manner. The diameter of the fungal colony was measured at the $6 \mathrm{~h}$ interval. Sterile coverslips were inserted into the agar medium at an angle of $45^{\circ}$, and the number of branching mycelia on the coverslip was scored using a microscope. To examine the number of spores on the medium, all mycelia growing on the surface of the medium were transferred to $10 \mathrm{~mL}$ PBS. The medium was violently shaken, and $100 \mu \mathrm{L}$ of the medium was transferred to the hemocytometer to count the spores under a microscope.

\section{Germ-free and gnotobiotic flies}

The process of generating germ free (GF) embryos was performed similar to that previously described $[15,23]$. Eggs laid on grape juice agar media were collected within $8 \mathrm{~h}$ and rinsed with $\mathrm{ddH} 2 \mathrm{O}$ to remove the yeast paste on the surface. Next, the eggs were sequentially subjected to 1:30 diluted Walch sanitizer (Procter \& Gamble Co., Cincinnati, OH, USA), 2.5\% hypochlorite sodium for 1 min (Sigma Aldrich, St. Louis, MO, USA), $70 \%$ ETOH for $1 \mathrm{~min}$, and finally PBS containing 0.01\% TritonX-100. The absence of bacteria was routinely confirmed by PCR analysis of $16 \mathrm{~S}$ rRNA primers (8FE and $1492 \mathrm{R})$ on fly homogenates and culturing the homogenates in Lysogeny Broth (LB)-agar plates. Sterilized GF eggs were transferred to vials with autoclaved media within a biosafety cabinet. GF flies were supplemented with either unknown or known bacteria to generate conventionally reared $(\mathrm{CR})$ or gnotobiotic flies.

\section{Survival rate and developmental timing of flies}

For the survival test, 30 eggs were transferred to vials with casein-cornmeal-agar medium within $10 \mathrm{~h}$ after egglaying. Eggs were exposed to fungi or bacteria, and the survival ratio was calculated. The number of pupae and adult formation was recorded, and the formula used to calculate developmental timing was expressed as:

$$
\mathrm{T}=(\mathrm{T} 1 \times \mathrm{N} 1+\mathrm{T} 2 \times \mathrm{N} 2+\ldots+\mathrm{Tm} \times \mathrm{Nm}) / \quad(\mathrm{N} 1+\mathrm{N} 2+
$$

... $+\mathrm{Nm})$.

where $T$ represents the developmental timing, $T_{m}$ is the days after egg laying, and $\mathrm{N}_{\mathrm{m}}$ is the number of pupae and adults on the $\mathrm{T}_{\mathrm{m}}$ day $[15,23]$.

\section{Oral and injury infection}

For survival assay, 20 male and female flies following $5 \mathrm{~d}$ after eclosion were collected into each vial. For oral infection, flies were transferred to vials that were preinoculated with $10^{8}$ spores and incubated at $25^{\circ} \mathrm{C}$ for 48 h. Systemic infections (septic injury) were performed by pricking the thorax of adult females with a thin needle previously dipped into a concentrated pellet of bacterial culture or into a suspension of Diaporthe FY spores [45]. All of the infected flies were incubated at $25^{\circ} \mathrm{C}$. At least three tubes containing 20 flies were used for survival experiments and the survival count was scored daily.

\section{Real-time quantitative PCR analysis}

Male adult flies were fed Diaporthe FY for $24 \mathrm{~h}$. Fly guts were dissected in cold PBS buffer, and the total RNA was extracted with TRIzol reagent (Invitrogen, USA). Up to $2 \mathrm{mg}$ of total RNA was used as a template for reverse transcription with the oligo-dT primer for real-time quantitative PCR (BioRad). The primer sets for ATT, Dpt, and Duox were as previously described [35]. The $\Delta \mathrm{Ct}$ method was employed to analyze the data using $r p 49$ as the reference gene. The relative expression value was calculated with formula: $\Delta \mathrm{Ct}=\mathrm{Ct}$ (target gene) $-\mathrm{Ct}$ (reference gene), the relative $=2^{-\Delta \Delta C t}$.

\section{Oviposition preference assay}

Two-choice oviposition chambers were constructed in a similar manner as described in previous studies [23, 28]. In each chamber, the flies were able to choose their oviposition sites between two types of fermented substrates. To create the fermented substrates, food agar was sterilized by autoclaving at $121^{\circ} \mathrm{C}$, and plated with either $100 \mu \mathrm{L}$ of Diaporthe FY, L. plantarum, or $\mathrm{dd}_{2} \mathrm{O}$ for the controls. Flies were then incubated at $25^{\circ} \mathrm{C}$ for $48 \mathrm{~h}$. To assemble the oviposition chamber, a razor blade was used to divide the agar into halves, and two different oviposition substrates were hand-puzzled into a Petri dish. To assess the fungal metabolites, liquid fly food (without agar) was inoculated with Diaporthe FY and incubated at $25^{\circ} \mathrm{C}$ for $48 \mathrm{~h}$. Fermented fly food was centrifuged at $12,000 \mathrm{rpm}$ for $15 \mathrm{~min}$, and the supernatant was distributed onto the surface of fly food in each of the two-choice oviposition chambers. A total of 50 female flies were collected and mated for $6 \mathrm{~h}$ after transfer to the device. Finally, the flies were removed and the number of eggs on 
each half of the two-choice chamber was counted, and the oviposition index (OI) was calculated using the following equation: $\mathrm{OI}=$ (no. of eggs laid on experimental food - no. of eggs laid on control food)/total no. of eggs laid.

\section{Supplementary information}

Supplementary information accompanies this paper at https://doi.org/10. 1186/s12866-019-1686-1.

Additional file 1. Pathogenic fungi undermine the fitness of Drosophila. Additional file 2. L. lactobacter generates lactic acid overtime.

Additional file 3. Modest $\mathrm{pH}$ decrease exhibits a trivial role in inhibiting the growth of Diaporthe FY.

\section{Abbreviations}

CFU: Colony formation unit; CR: Conventionally reared; GF: Germ free; Ol: Oviposition index

\section{Acknowledgements}

We would like to thank Alice Tan, Department of Biological Sciences, National University of Singapore for proof-reading the manuscript, and all members of the Liu Wei's lab for helpful discussion and criticism of this manuscript.

\section{Authors' contributions}

$W L, W S$, and $J$ conceived and designed the experiments; WS, JL, PB and BM performed the experiments; WS performed the statistical analysis; WL and WS wrote the paper. All authors read and approved the final manuscript.

\section{Funding}

This work was supported by the National Natural Science Foundation of China (31501175), and Grants from Shanxi Medical University Fenyang College (2018C02 and 2016D04). Funding bodies had no roles in study design and collection, analysis, and interpretation of data, or preparation of the manuscript.

\section{Availability of data and materials}

The dataset for the current study is available from the corresponding author upon reasonable request. L. plantarum was isolated from the gut of Drosophila with the Genbank accession number: KY038178.

\section{Ethics approval and consent to participate}

Not applicable.

\section{Consent for publication}

Not applicable.

\section{Competing interests}

The authors declare that they have no competing interests.

\section{Author details}

${ }^{1}$ Department of Clinical Medical, Shanxi Medical University Fenyang College, Fenyang 032200, Shanxi, China. ${ }^{2}$ Department of Basic Medical, Shanxi Medical University Fenyang College, Fenyang 032200, Shanxi, China. ${ }^{3}$ Department of Medical Laboratory Science, Shanxi Medical University Fenyang College, Fenyang 032200, Shanxi, China.

Received: 30 June 2019 Accepted: 15 December 2019

Published online: 21 December 2019

\section{References}

1. Charbonneau MR, Blanton LV, DiGiulio DB, Relman DA, Lebrilla CB, Mills DA, Gordon II. A microbial perspective of human developmental biology. Nature. 2016:535(7610):48-55.

2. Lee WJ, Brey PT. How microbiomes influence metazoan development: insights from history and Drosophila modeling of gut-microbe interactions. Annu Rev Cell Dev Biol. 2013;29:571-92.
3. Rees T, Bosch T, Douglas AE. How the microbiome challenges our concept of self. PLoS Biol. 2018;16(2):e2005358.

4. Rosenberg E, Zilber-Rosenberg I. The hologenome concept of evolution after 10 years. Microbiome. 2018;6(1):78.

5. Adair KL, Wilson M, Bost A, Douglas AE. Microbial community assembly in wild populations of the fruit fly Drosophila melanogaster. ISME J. 2018;12(4):959-72.

6. Chandler JA, Lang JM, Bhatnagar S, Eisen JA, Kopp A. Bacterial communities of diverse Drosophila species: ecological context of a host-microbe model system. PLoS Genet. 2011;7(9):e1002272.

7. Markow TA. The secret lives of Drosophila flies. Elife. 2015;4:e06793.

8. Caballero Ortiz S, Trienens M, Rohlfs M. Induced fungal resistance to insect grazing: reciprocal fitness consequences and fungal gene expression in the Drosophila-Aspergillus model system. PLoS One. 2013;8(8):e74951.

9. Peay KG, Kennedy PG, Talbot JM. Dimensions of biodiversity in the earth mycobiome. Nat Rev Microbiol. 2016;14(7):434-47.

10. Wang C, Wang S. Insect pathogenic Fungi: genomics, molecular interactions, and genetic improvements. Annu Rev Entomol. 2017;62:73-90.

11. Moonjely S, Barelli L, Bidochka MJ. Insect pathogenic Fungi as Endophytes. Adv Genet. 2016;94:107-35.

12. Raman A, Wheatley W, Popay A. Endophytic fungus-vascular plant-insect interactions. Environ Entomol. 2012:41(3):433-47.

13. latsenko I, Boquete JP, Lemaitre B. Microbiota-derived lactate activates production of reactive oxygen species by the intestinal NADPH oxidase Nox and shortens Drosophila lifespan. Immunity. 2018;49(5):929-42 e925.

14. Lemaitre B, Miguel-Aliaga I. The digestive tract of Drosophila melanogaster. Annu Rev Genet. 2013;47:377-404.

15. Shin SC, Kim SH, You H, Kim B, Kim AC, Lee KA, Yoon JH, Ryu JH, Lee WJ. Drosophila microbiome modulates host developmental and metabolic homeostasis via insulin signaling. Science. 2011;334(6056):670-4.

16. Trinder M, Daisley BA, Dube JS, Reid G. Drosophila melanogaster as a highthroughput model for host-microbiota interactions. Front Microbiol. 2017;8:751.

17. Storelli G, Defaye A, Erkosar B, Hols P, Royet J, Leulier F. Lactobacillus plantarum promotes Drosophila systemic growth by modulating hormonal signals through TOR-dependent nutrient sensing. Cell Metab. 2011;14(3):403-14.

18. Matos RC, Schwarzer M, Gervais H, Courtin P, Joncour P, Gillet B, Ma D, Bulteau AL, Martino ME, Hughes S, et al. D-Alanylation of teichoic acids contributes to Lactobacillus plantarum-mediated Drosophila growth during chronic undernutrition. Nat Microbiol. 2017;2(12):1635-47.

19. Schretter CE, Vielmetter J, Bartos I, Marka Z, Marka S, Argade S, Mazmanian SK. A gut microbial factor modulates locomotor behaviour in Drosophila. Nature. 2018;563(7731):402-6.

20. Blum JE, Fischer CN, Miles J, Handelsman J. Frequent replenishment sustains the beneficial microbiome of Drosophila melanogaster. MBio. 2013;4(6): e00860-13.

21. Dissanayake AJ, Liu M, Zhang W, Chen Z, Udayanga D, Chukeatirote E, Li X, Yan J, Hyde KD. Morphological and molecular characterisation of Diaporthe species associated with grapevine trunk disease in China. Fungal Biol. 2015; 119(5):283-94.

22. Moleleki N, van Heerden SW, Wingfield MJ, Wingfield BD, Preisig O. Transfection of Diaporthe perjuncta with Diaporthe RNA virus. Appl Environ Microbiol. 2003:69(7):3952-6.

23. Liu W, Zhang K, Li Y, Su W, Hu K, Jin S. Enterococci mediate the Oviposition preference of Drosophila melanogaster through sucrose catabolism. Sci Rep. 2017;7(1):13420

24. Ramirez-Camejo LA, Torres-Ocampo AP, Agosto-Rivera JL, Bayman P. An opportunistic human pathogen on the fly: strains of Aspergillus flavus vary in virulence in Drosophila melanogaster. Med Mycol. 2014;52(2):211-9.

25. Lemaitre B, Kromer-Metzger E, Michaut L, Nicolas E, Meister M, Georgel P, Reichhart JM, Hoffmann JA. A recessive mutation, immune deficiency (imd), defines two distinct control pathways in the Drosophila host defense. Proc Natl Acad Sci U S A. 1995:92(21):9465-9.

26. Storelli G, Strigini M, Grenier T, Bozonnet L, Schwarzer M, Daniel C, Matos R, Leulier F. Drosophila perpetuates nutritional mutualism by promoting the fitness of its intestinal Symbiont Lactobacillus plantarum. Cell Metab. 2018; 27(2):362-77 e368

27. Seddik HA, Bendali F, Gancel F, Fliss I, Spano G, Drider D. Lactobacillus plantarum and its probiotic and food potentialities. Probiotics Antimicrob Proteins. 2017:9(2):111-22

28. Joseph RM, Devineni AV, King IF, Heberlein U. Oviposition preference for and positional avoidance of acetic acid provide a model for competing behavioral drives in Drosophila. Proc Natl Acad Sci U S A. 2009;106(27):11352-7. 
29. Yang $\mathrm{CH}$, Belawat $\mathrm{P}$, Hafen $\mathrm{E}$, Jan $\mathrm{LY}$, Jan $\mathrm{YN}$. Drosophila egg-laying site selection as a system to study simple decision-making processes. Science. 2008;319(5870):1679-83.

30. Kempken F. Fungal defences against animal antagonists - lectins \& more. Mol Ecol. 2011;20(14):2876-7.

31. Sousa JP, Aguilar-Perez MM, Arnold AE, Rios N, Coley PD, Kursar TA, CubillaRios L. Chemical constituents and their antibacterial activity from the tropical endophytic fungus Diaporthe sp. F2934. J Appl Microbiol. 2016; 120(6):1501-8.

32. Sharon G, Sampson TR, Geschwind DH, Mazmanian SK. The central nervous system and the gut microbiome. Cell. 2016;167(4):915-32.

33. Chandler JA, Eisen JA, Kopp A. Yeast communities of diverse Drosophila species: comparison of two symbiont groups in the same hosts. Appl Environ Microbiol. 2012;78(20):7327-36.

34. Erkosar B, Storelli G, Mitchell M, Bozonnet L, Bozonnet N, Leulier F. Pathogen virulence impedes Mutualist-mediated enhancement of host juvenile growth via inhibition of protein digestion. Cell Host Microbe. 2015; 18(4):445-55.

35. Ryu JH, Kim SH, Lee HY, Jin YB, Nam YD, Bae JW, Dong GL, Shin SC, Ha EM, Lee WJ. Innate immune homeostasis by the Homeobox gene caudal and commensal-gut mutualism in Drosophila. Science. 2008;319(5864):777-82.

36. Westfall S, Lomis N, Prakash S. Longevity extension in Drosophila through gut-brain communication. Sci Rep. 2018;8(1):8362.

37. Gilliland SE. Antagonistic action of Lactobacillus acidophilus toward intestinal and foodborne pathogens in associative cultures. The host and its micronflora : an ecological unit. J Food Prot. 1977;40(12):820-3.

38. Russo P, Fares C, Longo A, Spano G, Capozzi V. Lactobacillus plantarumwith broad antifungal activity as a protective starter culture for bread production. Foods. 2017;6(12):110.

39. Chiocchetti GM, Jadan-Piedra C, Monedero V, Zuniga M, Velez D, Devesa V. Use of lactic acid bacteria and yeasts to reduce exposure to chemical food contaminants and toxicity. Crit Rev Food Sci Nutr. 2019;59(10):1534-45.

40. Christiaens JF, Franco LM, Cools TL, De Meester L, Michiels J, Wenseleers T, Hassan BA, Yaksi E, Verstrepen KJ. The fungal aroma gene ATF1 promotes dispersal of yeast cells through insect vectors. Cell Rep. 2014;9(2):425-32.

41. Kacsoh BZ, Lynch ZR, Mortimer NT, Schlenke TA. Fruit flies medicate offspring after seeing parasites. Science. 2013:339(6122):947-50.

42. Laturney M, Billeter JC. Neurogenetics of female reproductive behaviors in Drosophila melanogaster. Adv Genet. 2014;85:1-108.

43. Kurz CL, Charroux B, Chaduli D, Viallat-Lieutaud A, Royet J. Peptidoglycan sensing by octopaminergic neurons modulates Drosophila oviposition. Elife. 2017;6:e21937.

44. Stensmyr MC, Dweck HK, Farhan A, Ibba I, Strutz A, Mukunda L, Linz J, Grabe V, Steck K, Lavista-Llanos S. A conserved dedicated olfactory circuit for detecting harmful microbes in Drosophila. Cell. 2012;151(6):1345.

45. Lionakis MS, Kontoyiannis DP. Drosophila melanogaster as a model organism for invasive aspergillosis. Methods Mol Biol. 2012;845:455-68.

\section{Publisher's Note}

Springer Nature remains neutral with regard to jurisdictional claims in published maps and institutional affiliations.

Ready to submit your research? Choose BMC and benefit from:

- fast, convenient online submission

- thorough peer review by experienced researchers in your field

- rapid publication on acceptance

- support for research data, including large and complex data types

- gold Open Access which fosters wider collaboration and increased citations

- maximum visibility for your research: over $100 \mathrm{M}$ website views per year

At BMC, research is always in progress.

Learn more biomedcentral.com/submissions 